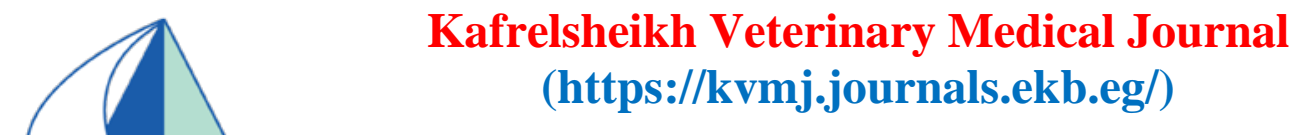

Original Article
CrossMark

Received July 12, 2021

Accepted July 27, 2021

Published October 15, 2021

\title{
Sequential proliferative lesions in the cirrhotic liver of rats: a histopathological and immunohistochemical study
}

\author{
Marwa Darweish*, Mahmoud GabAllh, AbdEl-baset El-Mashad, Shawky Moustafa, Aziza Amin \\ Department of Pathology, Faculty of Veterinary Medicine, Benha University, Egypt. \\ *Correspondence: marwayazeed3010@gmail.com
}

\begin{abstract}
Background/aim: Liver cirrhosis is a common health problem that is mostly at the late stage associated with hepatic carcinogenesis. This study aimed to clarify the sequential proliferative lesions in the cirrhotic liver induced chemically by thioacetamide (TAA) in rats.

Methods: Thirty male albino rats were equally divided into control and TAA groups. In the TAA group, rats were intraperitoneally injected with $100 \mathrm{mg} / \mathrm{kg}$ TAA twice /week for 18 weeks. Animals were euthanized after 6, 12, and 18 weeks. Blood and liver samples were collected for biochemical analysis and histopathological examination.

Results: TAA-treated group had significantly increased serum levels of liver damage parameters (alanine transaminase, aspartate transaminase, and alkaline phosphatase), and hepatic levels of oxidative stress-related markers (malondialdehyde and nitric oxide) as compared to the control group. Meanwhile, the TAA group showed significantly decreased hepatic levels of antioxidant markers (reduced glutathione and superoxide dismutase) relative to the control group. Histopathological examination of the liver revealed characteristic lesions of liver cirrhosis in form of regenerative nodules, the proliferation of bile ducts epithelium and hepatic stellate cells as well as different types of foci of cellular alterations which were prominent proliferative lesions in the liver of the TAA group. However, with TAA prolonged treatment, cellular atypia and preneoplastic changes became evident in many hepatocytes. Immunohistochemical expression for Ki67 revealed positive nuclear labeling in some hepatocytes of TAA-treated rats. Marked expression for Ki67 in regenerating nodules at 18 weeks following TAA treatment was interesting as it revealed a higher regeneration activity.

Conclusion: Some proliferative lesions such as regenerative nodules and foci of cellular alteration in hepatic cirrhosis may later constitute carcinogenic development.
\end{abstract}

Keywords: TAA; liver; cirrhosis; histopathology; ki67

\section{Introduction}

Liver is a vital organ responsible for the metabolism, bile secretion, elimination of many substances, and blood detoxifications (Alshawsh et al., 2011). Liver fibrosis is a major health problem that results in significant morbidity and mortality (Sa'nchez-Valle et al., 2012). The pathogenesis of liver fibrosis is complex and is characterized by the deposition of extracellular matrix (ECM) by activated hepatic stellate cells (HSC) (Massimo and Macias-Barragan, 2014). Thioacetamide (TAA) is a thionosulfur-containing compound, which is widely used to induce liver fibrosis in rats to investigate the underlying mechanisms and therapeutic effects of potential anti-fibrotic drugs (Atef et al., 2016).

Hepatic cirrhosis is a progressive consequence of liver fibrosis. Recently, more intensive studies within the pathology community have been performed on liver fibrosis, however, few reports are describing a variety of non-neoplastic proliferative lesions associated with liver cirrhosis and may be induced by treatment with chemicals. Some of these lesions might be regarded as pre-neoplastic alterations. It was documented that high-grade dysplastic nodules have a risk of hepatocarcinogenesis; however, no fear from large regenerative nodules for malignant transformation (Libbrecht et al., 2005). Detailed knowledge of early changes involving these preneoplastic lesions is an important preliminary study that could open up a new therapy to arrest the development of hepatocellular carcinomas associated with liver cirrhosis. Therefore, controlling fibrosis and preventing the progression of hepatic cirrhosis is important for preventing the development of sequelae such as hepatocellular carcinoma (Gines et al., 2004; Coombes et al., 2015a; Coombes et al., 2015b).

Therefore, the present study aimed to clarify the sequential proliferative lesions of the cirrhotic liver in rats via histopathological and immunohistochemical assessment. 


\section{Materials and methods}

\subsection{Experimental design}

Thirty male albino rats (120-150 g) obtained from the research laboratory of the Faculty of Veterinary Medicine, Zagazig University, Egypt were housed in cages in a well-ventilated house and allowed ad libitum access to food and water and kept in a $12 \mathrm{hr}$ light-dark cycle. All the animals that received human care according to standard guidelines were randomly divided into two equal groups ( $\mathrm{n}=15$ /group). In the control group, rats were intraperitoneally injected with $0.5 \mathrm{ml}$ saline twice / week for 18 weeks. In the TAA group, rats were intraperitoneally injected with $100 \mathrm{mg} / \mathrm{kg}$ TAA (0.5 ml per rat), twice /week for 18 weeks according to Hamed et al., (2011). TAA was purchased from (Sigma-Aldrich, St. Gallen, Switzerland) in form of a white crystalline solid which was dissolved in saline. After 6, 12, and 18 weeks, rats were fasted for $8 \mathrm{~h}$, weighed, anesthetized by light ether then euthanized by cervical dislocation. Blood and liver samples were collected from five rats of each timepoint $(6,12$, and 18 weeks) for each group for biochemical analysis and histopathological examination.

\subsection{Biochemical analysis}

Liver function parameters including alanine transaminase (ALT, Diamond- Diagnostics, Egypt, Cat. No. 264001), aspartate transaminase (AST, Cat. No. 261004), and alkaline phosphatase (ALP, Cat. No. 241002) were measured in serum. Malondialdehyde (MDA, Biodiagnstic, Egypt, Cat. No. 2529), nitric oxide (NO) (Cat. No. 2533), reduced glutathione (GSH, Cat. No. 2511), and superoxide dismutase (SOD, Cat. No. 2521) in liver homogenate for detection of oxidative stress and antioxidant parameters. The liver homogenate was prepared as previously described (El-Bayomi et al., 2018).

\subsection{Histopathological examination}

Liver specimens were preserved in $10 \%$ neutral buffered formalin. After proper fixation, the livers were trimmed, washed in running tap water, dehydrated in different ascending concentrations of ethanol, and cleared in xylene. Paraffin blocks were prepared, then sectioned at $5 \mu \mathrm{m}$ by a rotary microtome and stained with hematoxylin and eosin (H\&E)and Masson's trichrome stain (Bancroft et al., 1996).

\subsection{Immunohistochemistry}

Paraffin sections were prepared into positive slides and processed for immunohistochemical examination using a streptavidin-biotinperoxidase method according to Ramos-Vara et al., (2008). The technique was performed using primary antibodies for ki67 (Abcam; ab16667; 1:100).

\subsection{Statistical Analysis}

Statistical analysis of the results was carried out using the student's $t$-test. Data were presented as mean $\pm \mathrm{SEM}$. Significance was set at $\mathrm{P}<0.05$.

\section{Results}

\subsection{Biochemical results}

The TAA-treated group had significantly $(\mathrm{P}<0.001)$ increased
ALT, AST, and ALP levels as compared to the control group (Table 1). This elevation in liver enzyme activity was time-dependent, reaching their highest levels after 18 weeks of treatment. The concentrations of MDA and NO as markers of oxidative stress in the liver of the TAA group showed a significant $(\mathrm{P}<0.001)$ increase in the liver compared to the control group (Table 2). However, hepatic levels of the antioxidant markers GSH, and SOD showed a significant ( $\mathrm{P}<0.001)$ decrease relative to the control group. The changes in oxidative stress markers and antioxidant enzymes were also timedependent.

\subsection{Pathological results}

The livers of control rats grossly appeared normal, with a smooth surface. While the livers of TAA-treated rats were tan and showed multifocal to coalescing micro-nodules which replaced the whole hepatic surface at 18 weeks after TAA treatment (Fig. 1A). No microscopical findings were observed in the livers of control rats. In contrast, livers of TAA-treated rats after 6 weeks showed variable amounts of fibrous tissue proliferation infiltrated by few macrophages-laden brownish pigments within the cytoplasm in the portal areas (Fig. 2A). Occasionally, portal-to-portal bridging was observed, which is composed of mild to moderate fibrosis and marked biliary hyperplasia. Rarely, there were multifocal, various sizes, regenerative nodules replaced the normal hepatic architecture and were encircled by marked fibrosis (Fig. 2A). The proliferated fibrous tissue exhibited marked hyperplasia of biliary epithelium and stellate cells intermixed with few inflammatory cells (Fig. 2B). Whereas, large eosinophilic foci of cellular alteration were rarely observed (Fig. 2C). These foci were non-neoplastic, a localized proliferation of hepatocytes; the portal areas and central veins were rare or absent in the foci. The hepatic cells within the foci were enlarged, polygonal, with pale pink cytoplasm, enlarged nucleus, and prominent centrally located nucleolus (Fig. 2D).

The microscopical examination of the livers after 12 weeks of treatment with TAA revealed disruption of the hepatic parenchyma with marked portal fibrosis. The characteristic lobular pattern of the liver was displaced by variable-sizes regenerative nodules (Fig. 3A). Marked portal-to-portal bridging was observed. Regenerative nodules were encircled by marked fibrosis. Moderate numbers of hepatocytes within regenerative nodules exhibited marked hydropic degeneration characterized by swollen, pale, vacuolated cytoplasm (Fig. 3B). Multifocally, the portal area was expanded by bile duct hyperplasia with the formation of new nests of bile ducts surrounded by fibroblasts, stellate cells, and few lymphocytes (Fig. 3C). Fibrous tracts contained also moderate hyperplasia of biliary epithelium and stellate cells, occasionally intermixed with few aggregates of dissociated hepatocytes (Fig. 3D). There was a decrease in the number of portal areas, whereas few areas were recognized in fibrous tracts.

The livers of TAA-treated rats after 18 weeks showed disruption of the hepatic parenchyma with marked portal fibrosis (Fig. 4A). Marked portal-to-portal fibrosis was observed. Multifocally, fibrous tracts contained moderate hyperplasia of biliary epithelium and stellate cells that were occasionally intermixed with few aggregates of dissociated hepatocytes (Fig. 4B). Small infiltrates of mixed inflammatory cells were noticed in proliferated fibrous tissue. The characteristic lobular pattern of the liver was displaced by variablesizes regenerative nodules. Regenerative nodules were separated and surrounded by fine to moderate strands of fibrous connective tissue proliferation, occasionally expanded by edema intermixed with 
hyperplastic bile ductules and small infiltrates of mixed inflammatory cells. Predominantly within regenerative nodules, numerous hepatocytes exhibited normal appearance (Fig. 4C), however, many hepatocytes showed marked hepatocellular polyploidy and hepatocellular hypertrophy (Fig. 4D) with cytoplasmic hypereosinophilia, marked enlarged nuclei, often with prominent nucleoli and rare intra-nuclear inclusions (Fig. 5A); or foamy cytoplasm and small deeply basophilic nuclei (Fig. 5B). There was a decrease in the number of portal areas, whereas few areas were recognized in fibrous tracts. Multifocally, within regenerating nodules and proliferated fibrous tissue, the hepatocytes and Kupffer cells revealed brownish pigments within the cytoplasm. Rarely, there were foci of lytic necrosis with loss of hepatic architecture and replaced by eosinophilic necrotic debris, mixed with fibrin, and large numbers of lymphocytes and macrophages. On the other hand, large size clear cell foci of cellular alteration composed of hypertrophied hepatocytes were rarely observed (Fig. 5C). These foci were non-neoplastic, a localized proliferation of hepatocytes that merge imperceptibly with surrounding hepatic parenchyma without separation or surrounding fibrous connective tissue; the portal areas and central veins were rare or absent in the foci. The hepatic cells within the foci were enlarged, polygonal, with pale pink vacuolated cytoplasm, enlarged nucleus, and prominent centrally located nucleolus (Fig. 5D). Multifocally, there were moderate to marked bile duct hyperplasia in portal areas and within the fibrous tracts with multiple cystic or saccular dilatations lined by low cuboidal epithelial cells which forms branching papillary projections consisting of one row of cuboidal epithelium supported on a moderate fibro-vascular stroma.

\subsection{Immunohistochemical results}

Immunohistochemical stain for Ki67 in the examined liver was performed as a marker of cell proliferation. Liver of control rat showed very mild expression for Ki67 in few hepatocytes (Fig. 6A). Meanwhile, livers of TAA-treated rats after 6, 12, and 18 weeks showed positive expression for Ki67 in some hepatocyte nuclei (Fig. 6B, 6C, 6D). Low expression for Ki67 has been observed in the liver of TAA-treated rats after 6weeks (Fig. 6B) compared to higher expression in hepatocytes of TAA-treated rats after 12 weeks (Fig. 6C) and 18 weeks (Fig. 6D) of treatment. Marked expression for Ki67 was detected in regenerating nodules, characterized by intense brownish labeling of hepatocytes nuclei and an increase in numbers of positive cells (Fig. 6D).

Table 1: Effect of TAA on liver damage parameters

\begin{tabular}{|c|c|c|c|c|c|c|c|c|c|}
\hline \multirow[b]{2}{*}{ Group } & \multicolumn{3}{|c|}{ ALT(U/L) } & \multicolumn{3}{|c|}{ AST(U/L) } & \multicolumn{3}{|c|}{$\mathrm{ALP}(\mathrm{U} / \mathrm{L})$} \\
\hline & 6 Weeks & 12 Weeks & 18 Weeks & 6 Weeks & 12 Weeks & 18 Weeks & 6 Weeks & 12 Weeks & 18 Weeks \\
\hline Control & $27.81 \pm 2.87$ & $33.80 \pm 2.48$ & $29.80 \pm 2.43$ & $34.20 \pm 1.83$ & $32.00 \pm 1.70$ & $37.00 \pm 1.75$ & $78.60 \pm 1.44$ & $71.40 \pm 1.29$ & $76.40 \pm 1.36$ \\
\hline TAA & $221.20 \pm 15.30^{*}$ & $292.60 \pm 14.07^{*}$ & $296.60 \pm 14.11^{*}$ & $270.00 \pm 14.88^{*}$ & $324.00 \pm 17.87^{*}$ & $349.00 \pm 17.59^{*}$ & $277.40 \pm 7.45^{*}$ & $302.60 \pm 8.18^{*}$ & $336.20 \pm 8.97^{*}$ \\
\hline
\end{tabular}

Mean values with * in the same column are significantly different at $\mathrm{P}<0.001$. Data were presented as mean \pm SEM.

Table 2: Effect of TAA on oxidative stress/antioxidant status of the liver

\begin{tabular}{ccccccc}
\hline Group & \multicolumn{3}{c}{ MDA (nmol/g tissue) } & \multicolumn{3}{c}{ NO (umolg/g tissue) } \\
& 6 Weeks & 12 Weeks & 18 Weeks & 6 Weeks & 12 Weeks & 18 Weeks \\
\hline Control & $43.80 \pm 2.24$ & $40.80 \pm 2.24$ & $45.80 \pm 2.24$ & $38.60 \pm 1.50$ & $34.80 \pm 1.39$ & $37.00 \pm 1.30$ \\
TAA & $225.00 \pm 18.29^{*}$ & $255.00 \pm 18.29^{*}$ & $305.00 \pm 18.29^{*}$ & $89.20 \pm 2.56^{*}$ & $96.80 \pm 2.76^{*}$ & $103.00 \pm 3.03^{*}$ \\
\hline Group & 6 Weeks & GSH (mg/g tissue) & & & SOD (U/g tissue) \\
& $2.86 \pm 0.14$ & $3.09 \pm 0.15$ & $2.89 \pm 0.14$ & $42.60 \pm 1.29$ & $39.76 \pm 1.20$ & $43.38 \pm 1.31$ \\
\hline Control & $0.89 \pm 0.04^{*}$ & $0.82 \pm 0.04^{*}$ & $0.74 \pm 0.03^{*}$ & $23.40 \pm 1.86^{*}$ & $19.50 \pm 1.55^{*}$ & $16.72 \pm 1.33^{*}$ \\
TAA & & & & & & \\
\end{tabular}

Mean values with * in the same column are significantly different at $\mathrm{P}<0.001$. Data were presented as mean \pm SEM.
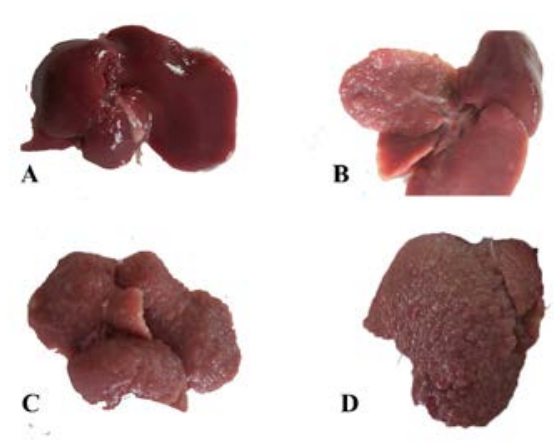

Fig. 1. Gross lesions of the liver. (A) The liver of the control group appeared normal with a smooth surface. (B-D) Livers of the TAA group show tan color and multifocal to coalescing micro-nodules; which were little after 6 weeks (B), moderate after 12 weeks (C), and marked after 18 weeks (D). 
(A)

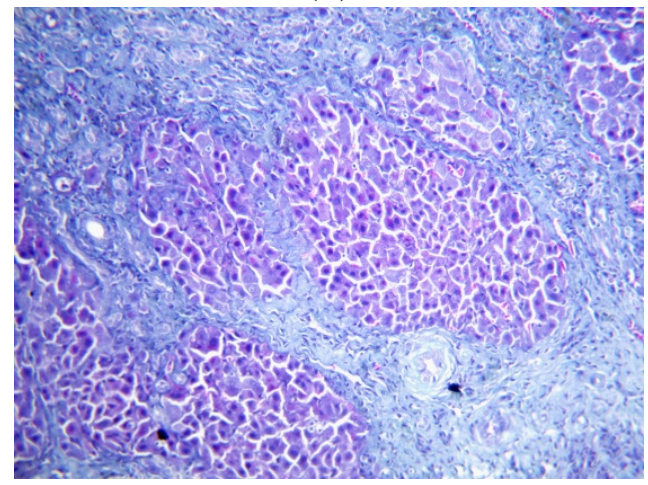

(C)

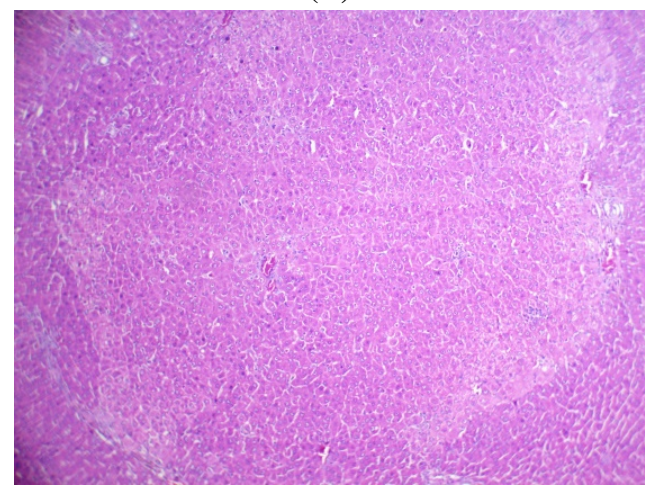

(B)

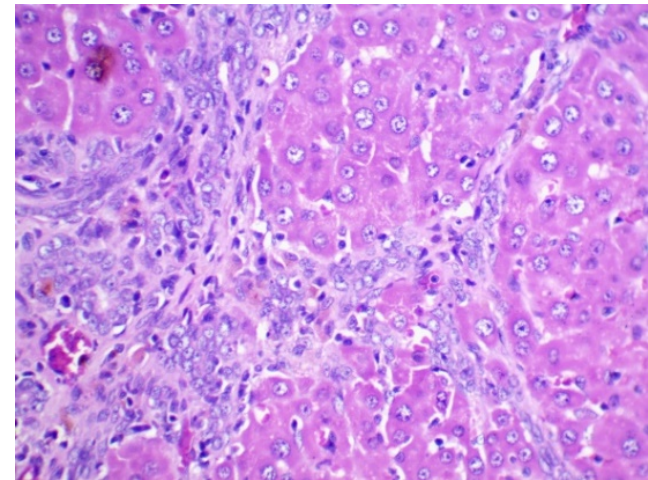

(D)

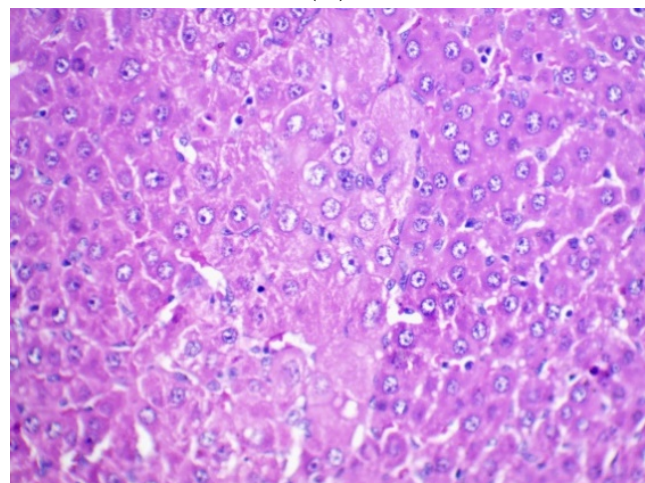

Fig 2: Liver of rats treated with TAA for 6 weeks. (A) Regenerative nodules replaced the normal hepatic architecture and encircled by marked fibrosis, Masson's trichrome stain x 200. (B) The proliferated fibrous tissue exhibited marked hyperplasia of the biliary epithelium (arrow) and stellate cells (arrowhead), H\&E stain x 400. (C) Large eosinophilic foci (EF) of cellular alteration, H\&E stain x 100 (D) High power of eosinophilic foci with enlarged, polygonal hepatocytes (arrow), pale pink cytoplasm, and mild enlarged nuclei H\&E stain x 400.

(A)

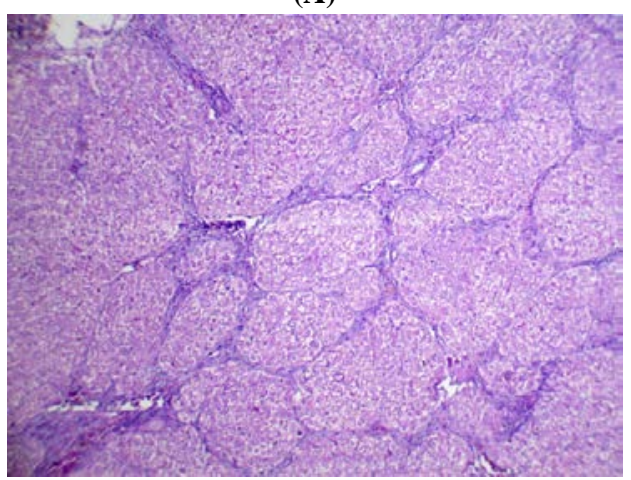

(C)

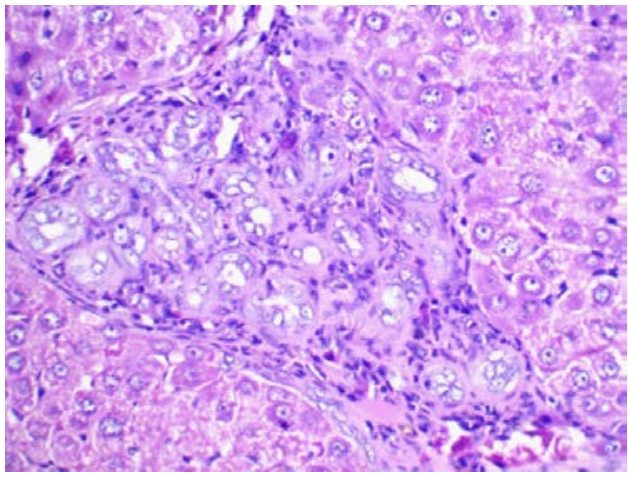

(B)

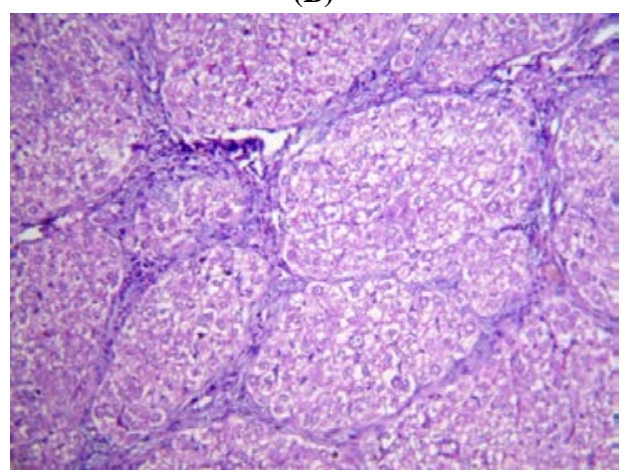

(D)

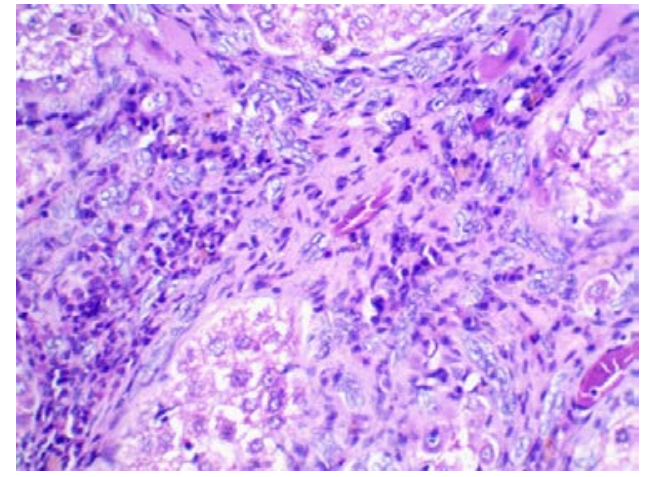

Fig 3. Liver of rat treated with Thioacetamide for 12 weeks, (A) Numerous, variable sizes, regenerative nodules (RN) replaced the normal hepatic architecture, H\&E stain x 100. (B) Hepatocytes within regenerative nodules exhibited marked hydropic degeneration (arrowhead), H\&E stain x 200. (C) Bile duct hyperplasia with the formation of new nests (arrow) surrounded by fibroblasts, and stellate cells, H\&E stain x 200. (D) Fibrous tracts contained moderate hyperplasia of the biliary epithelium (arrow) and stellate cells (arrowhead), H\&E stain x 200. 
(A)

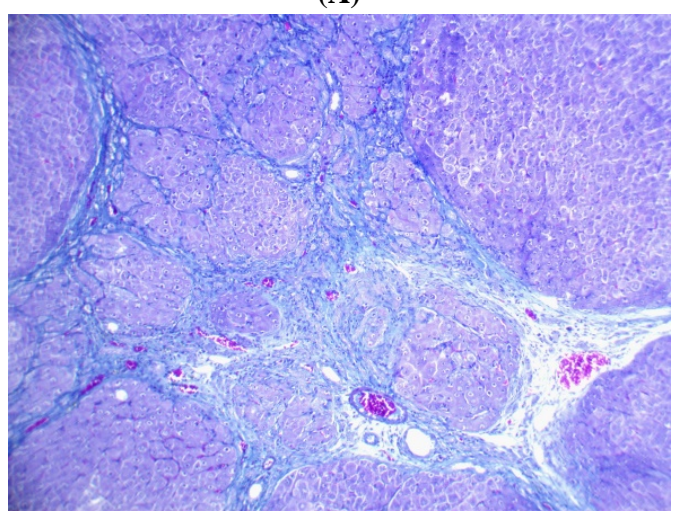

(C)

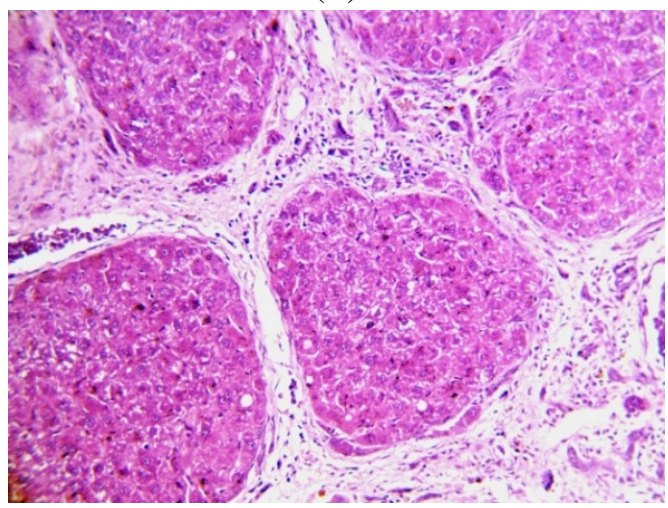

(B)

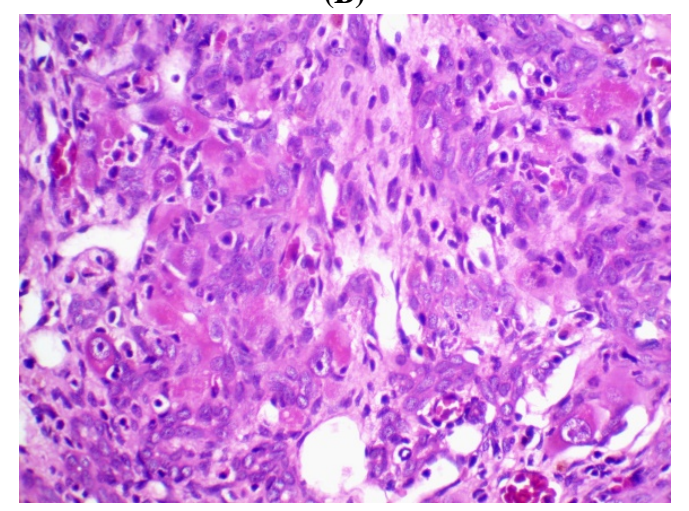

(D)

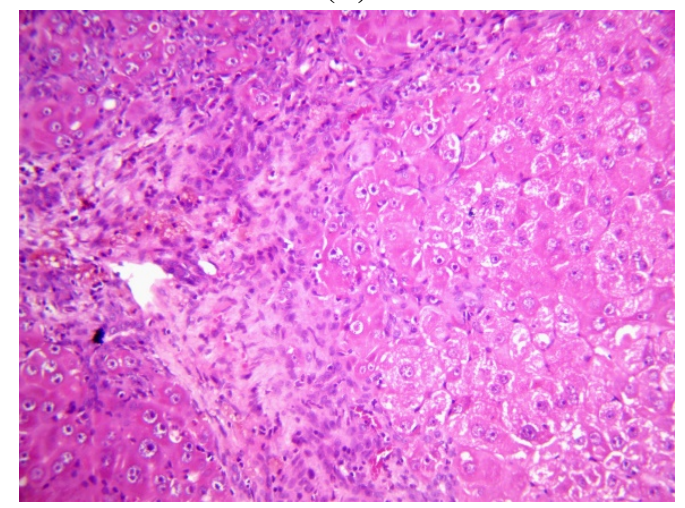

Fig 4. Liver of rat treated with Thioacetamide for $18 \mathrm{w}$. (A) Disruption of the hepatic parenchyma with marked portal fibrosis (F), Masson's trichrome stain $\mathrm{x} 100$ (B) Proliferation of bile ducts and stellate cells in fibrous tract intermixed with few aggregates of dissociated hepatocytes (arrow), H\&E stain x 400. (C) Regenerative nodules (RN), with normal appearance of hepatocytes and loss of normal lobule architecture, H\&E stain x 200. (D) Marked hepatocellular polyploidy (arrow) and hepatocellular hypertrophy within the regenerative nodule, H\&E stain x 200.

(A)

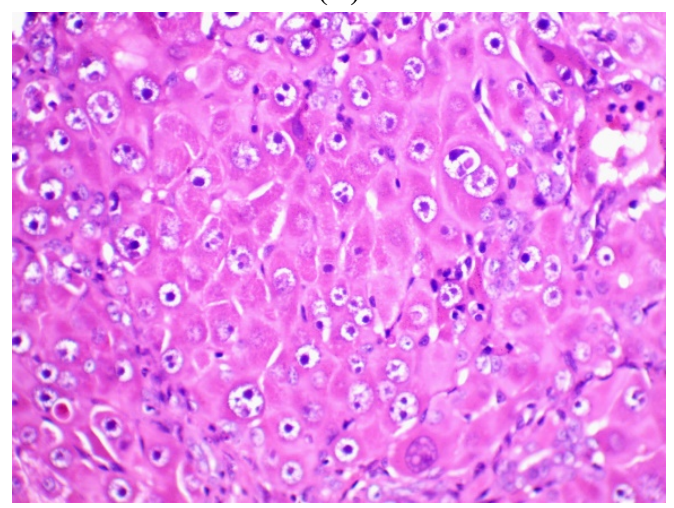

(C)

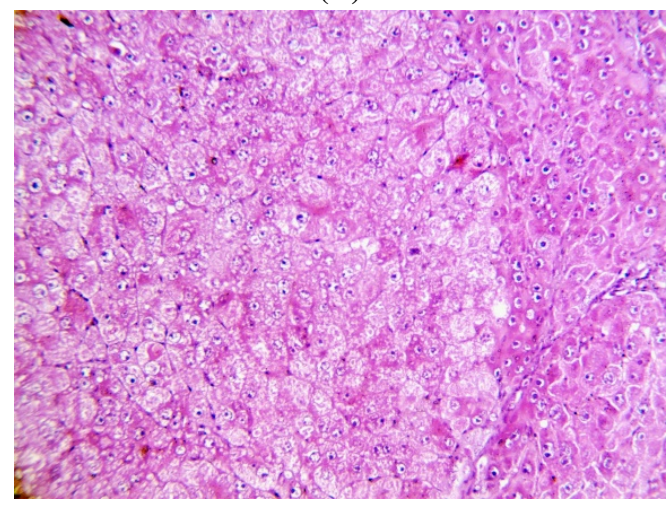

(B)

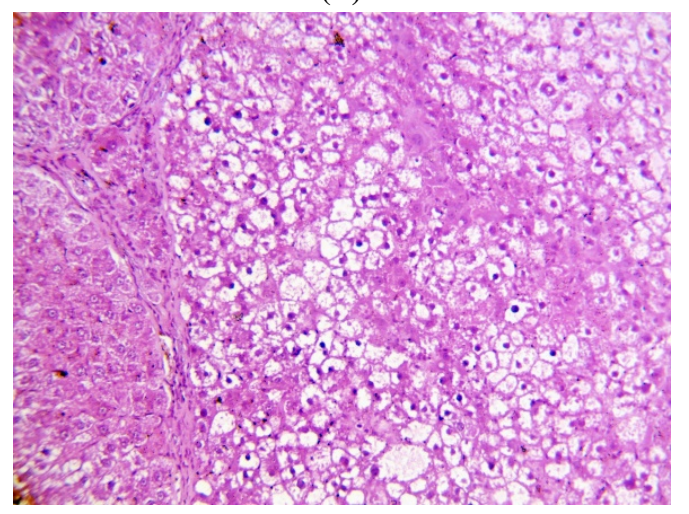

(D)

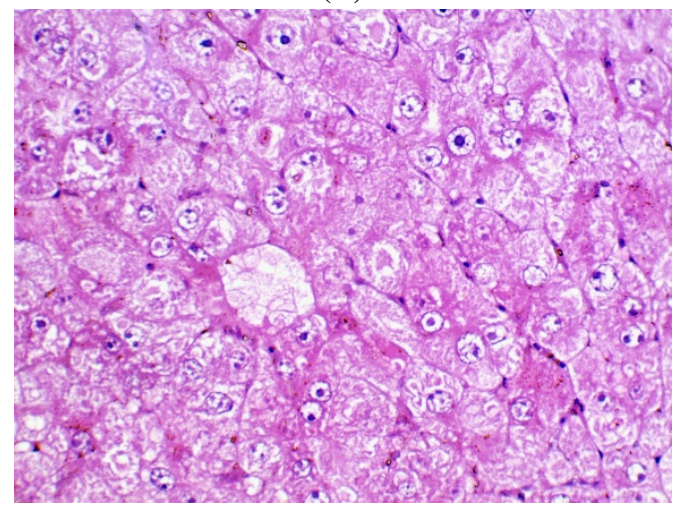

Fig 5. Liver of rat treated with Thioacetamide for 18 weeks. (A) Proliferated hepatocytes with cytoplasmic hypereosinophilia, marked enlarged nuclei, prominent nucleoli, and intranuclear inclusions (arrow), H\&E stain x 400. (B) Regenerative nodules contain enlarged hepatocytes with foamy cytoplasm and small deeply basophilic nuclei (arrowhead), H\&E stain x 200. (C) Clear cell foci (CF) of cellular alteration composed of hypertrophied hepatocytes, H\&E stain x 200. (D) High power of clear cell foci showed marked hepatocellular hypertrophy with pale pink vacuolated cytoplasm (arrow), enlarged nucleus, and prominent centrally located nucleolus, H\&E stain x 400 . 

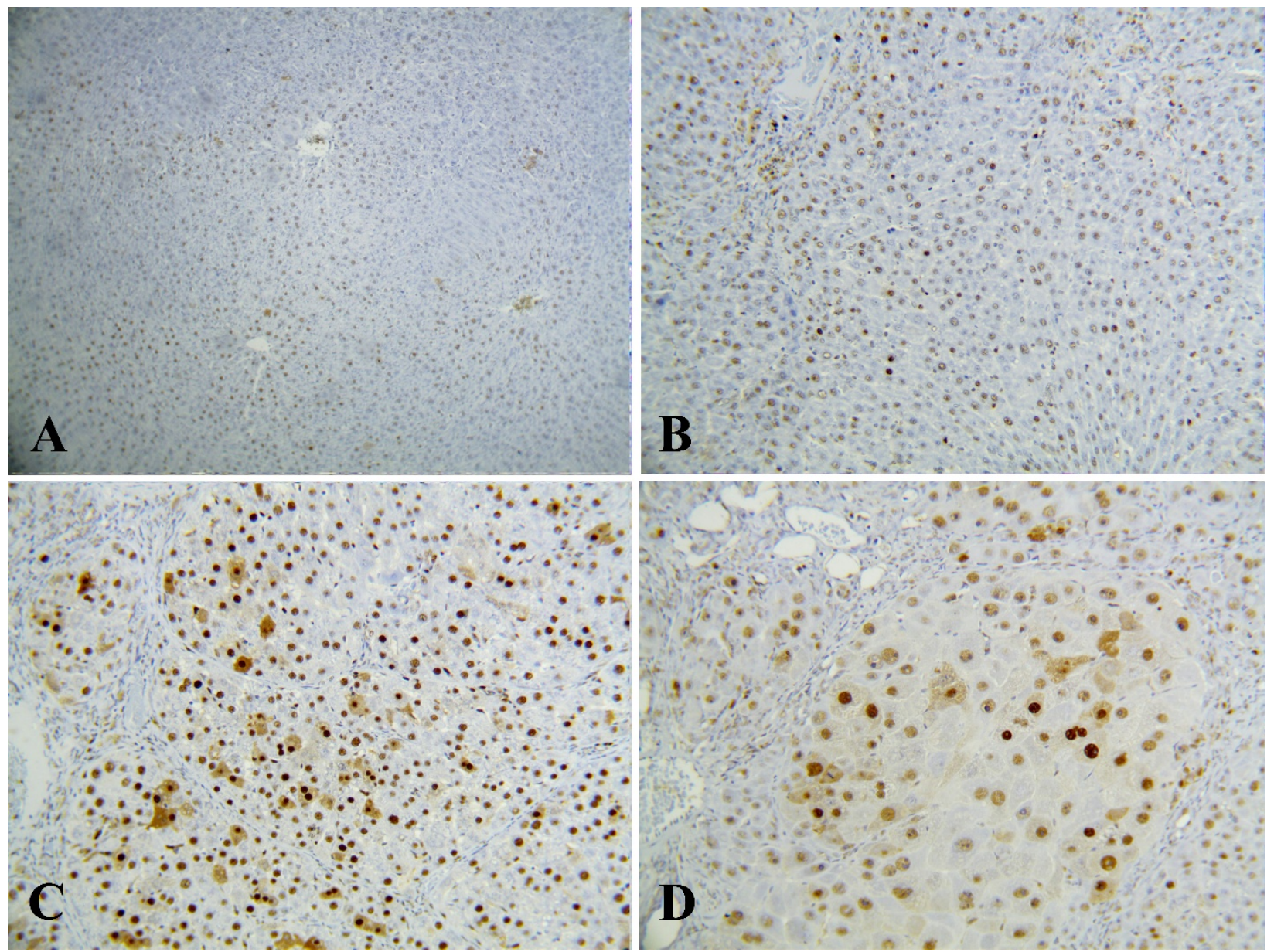

Fig. 6. Immunohistochemical stains for Ki67 in Rat Liver. (A) Liver of control rat showing very mild expression for Ki67, (x100). (B, C, D) livers of TAA-treated rats after 6, 12, and 18 weeks respectively, showing positive expression for Ki67 in some hepatocyte nuclei (x200). High expression in hepatocytes after 12 weeks (C) and 18 weeks (D) of TAA treatment compared with lower expression in the liver of TAA treated rats for 6weeks (B). Marked expression for Ki67 in regenerating nodules (D), characterized by intense brownish labeling of hepatocytes nuclei (arrowhead) and increase in numbers of positive cells.

\section{Discussion}

Chronic liver injury is characterized by activation of hepatic stellate cells with induction of fibrogenic repair and development of liver fibrosis (Coombes et al., 2015a; Coombes et al., 2015b). The present study revealed that TAA-treated rats had an abundant hepatotoxic effect, clearly demonstrated by a significant increase of hepatic enzyme activities (ALT, AST, ALP) in serum and oxidative stress marker (MDA, NO) in hepatic homogenate, but with a significant decrease in antioxidants GSH and SOD level. The metabolism of TAA involved the formation of a thiono-sulfur compound, which induce hepatic damage and fibrosis might responsible for these biochemical changes (Hajovsky et al., 2012; mousa et al., 2019; Zargar, 2014)

Grossly, the liver of TAA-treated rats showed multifocal to coalescing micro-nodules on hepatic surfaces. This lesion was commonly reported as a characteristic finding in liver cirrhosis (Amin et al., 2012; Hwang et al., 2012; Ramos-Tovar et al., 2019). Microscopically, there is disruption of the hepatic parenchyma with marked portal fibrosis, which was observed as early as 6 weeks post-TAA treatment. However, regenerative nodules replaced the normal hepatic architecture and encircled by marked fibrosis were a common proliferative lesion detected in the present study. Predominantly within regenerative nodules, numerous hepatocytes exhibited normal appearance, however, with prolonged treatment using TAA (18 weeks), cellular atypia become evident and many hepatocytes showed marked polyploidy and hypertrophy with cytoplasmic hypereosinophilia, marked enlarged nuclei, with prominent nucleoli and rare intra-nuclear inclusions. Unfortunately, there is some controversy about regenerative nodules as preneoplastic lesions in the cirrhotic liver. Previous studies recorded that there is no enough information about primitive hepatocarcinogenesis of large regenerative nodules (Libbrecht et al., 2005).

One of the characteristic proliferative lesions of the cirrhotic liver in this study is hyperplasia of biliary epithelial cells and hepatic stellate cells (HSC) that referred to as bi-potential progenitor cells. Hyperplasia of HSC induces the formation of ductular structures lined by cuboidal, basophilic cells. (jubb et al., 2016; Zimmermann et al., 2011). Interestingly, different types of foci of cellular alteration represent a focal proliferation of hepatocytes. The proliferated hepatocytes differ morphologically from normal hepatocytes of hepatic lobules that were observed in the current study in cirrhotic livers. Diagnosis of these foci is based on the predominant cell type present by differences of staining color, size of cells, and textural characters from normal hepatic parenchyma. There were two types; large eosinophilic and clear cell foci composed of hypertrophied hepatocytes; cellular atypia becomes evident in many hepatocytes. The relation between specific types of foci of cellular alteration and carcinogenesis has been previously documented (Cano et al., 2018).

Immunohistochemical results in this work revealed very mild 
expression for Ki67 in few hepatocytes of the control group which is in agreement with (Youssef et al., 2012), due to normal homeostasis turnover of the liver. Meanwhile, livers of TAAtreated rats have increased ki-67 expression than the control group and this expression was higher after 18 weeks of TAA treatment compared to expression in hepatocytes after 12 or 6 weeks. Interestingly, the findings of marked expression for Ki67 in regenerating nodules indicated a potent response to regeneration. Our results could be supported by (Arffa et al. 2016), who stated that livers of TAA intoxicated rats have increased ki-67 expression than treated groups. On contrary, (Clouston et al. 2005), recorded that the response of the liver for the regenerative process, with the formation of new hepatocytes, is inhibited in the presence of hepatic fibrosis compared to non-fibrotic livers. Although the current study revealed an increase in regenerating process in the case of liver fibrosis, the regenerating cells don't perform the function of normal hepatocytes. Excessive accumulation of extracellular matrix proteins in liver fibrosis could alter hepatic cytoarchitecture, leading to an obstruction of function (Bataller and Brenner 2005).

\section{Conclusion}

Our investigation on the cirrhotic liver in the rat model clarified different proliferative lesions such as regenerative nodules and foci of cellular alteration that may later constitute carcinogenic development. Further genetic and epigenetic studies may give a thorough light to a new therapy for hepato-carcinogenesis.

\section{Conflict of interest}

The authors declare that they have no conflict of interest.

\section{Research Ethics Committee Permission}

This study was approved by the local Ethics and guides of the Faculty of Veterinary Medicine Benha University Egypt (BUFVTM 01/11/2019).

\section{Authors' contribution}

The study is part of a $\mathrm{PhD}$ thesis presented to the Department of Pathology, Faculty of Veterinary Medicine, Benha University by $\mathrm{MD}$, where MG, AE, SM, and AA acted as thesis supervisors.

\section{References}

Alshawsh, M., Abdulla, M., Ismail, S., Amin, Z. 2011: Hepatoprotective effects of Orthosiphon stamineus extract on thioacetamide-induced liver cirrhosis in rats. Evidence-based complementary and alternative medicine

Amin, Z., Bilgen, M., Alshawsh, M., Ali, H., Hadi, A., Abdulla, M. 2012: Protective role of Phyllanthus niruri extract against thioacetamide induced liver cirrhosis in rat model. evidencebased complementary and alternative medicine

Arffa, M., Zapf, M., Kothari, A., Chang, V., Gupta, G., Ding, X., Al-Gayyar, M., Syn, W., Elsherbiny, N., Kuo, P., Mi, Z. 2016: Epigallocatechin-3-gallate upregulates miR-221 to inhibit osteopontindependent hepatic fibrosis. PLoS One, 11(12): e0167435.

Atef, M., Al-Attar, Hayfa, A., Al-Rethea. 2017: Chemoprotective effect of omega-3 fatty acids on thioacetamide induced hepatic fibrosis in male rats. Saudi Journal of Biological Sciences Volume 24, Issue 4, May 2017, Pages 956-965

Bancroft, J., Cook, H., Beckstead, J. 1996: Manual of histological techniques and their diagnostic application. Archives of Pathology and Laboratory Medicine 120: 986-986.

Bataller, R., Brenner, D. 2005: Liver fibrosis. J Clin Invest 115(2):209-218.

Cano, L., Cerapio, J., Ruiz, E. 2018: Liver clear cell foci and viral infection are associated with non-cirrhotic, non-fibro lamellaMr hepatocellular carcinoma in young patients from South America. Scientific reports, 8, 9945 | DOI:10.1038/s41598-018-28286-0 12

Clouston, A., Powell, E., Walsh, M., Richardson, M., Demetris, A., Jonsson, J. 2005: Fibrosis correlates with a ductular reaction in hepatitis C: roles of impaired replication, progenitor cells and steatosis. Hepatology, 41(4):809-18, 2005.

Coombes, J., Choi, S., Swiderska-Syn, M., Manka, P., Reid, D., Palma, E. 2015a: Osteopontin is a proximal effector of leptinmediated non-alcoholic steatohepatitis (NASH) fibrosis. Biochim Biophys Acta.

Coombes J., Swiderska-Syn, M., Dolle, L., Reid, D., Eksteen, B., Claridge, L. 2015b: Osteopontin neutralization abrogates the liver progenitor cell response and fibrogenesis in mice. Gut; 64(7):11201131.

El-Bayomi KM, Saleh AA, Awad A, El-Tarabany MS, El-Qaliouby HS, Afifi M, El-Komy S, Essawi WM, Almadaly EA, El-Magd MA. 2018: Association of Cyp19a1 gene polymorphisms with anoestrus in water buffaloes. Reproduction, Fertility and Development 30:487-497.

Gines, P., Cardenas, A., Arroyo, V., Rodes, J. 2004: Management of cirrhosis and ascites.

N Engl J Med 2004 Apr15; 350(16):1646-1654.

Hajovsky, H., Hu, G., Koen, Y., Sarma, D., Cui, W., Moore, D., Staudinger, J., Hanzlik, R. 2012: Metabolism and toxicity of thioacetamide and thioacetamide S-oxide in rat hepatocytes. Chemical research in toxicology 25: 1955-1963.

Hamed, G., Bahgat, N., Abdel Mottaleb, F., Emara, M. 2011: Effect of flavonoid quercetin supplement on the progress of liver cirrhosis in rats. Life Sci J 8: 64.651-1

Hwang, S., Hong, H., Kim, H., Park, S., Won, Y., Choi, S., Choi, D., Lee, S. 2012: Hepatogenic differentiation of mesenchymal stem cells in a rat model of thioacetamide-induced liver cirrhosis. Cell biology international 36: 279-288.

Jubb, K ., Kennedy, P., Palmer, N. 2016: Pathology of Domestic Animals. Edited by M. Grant Maxie, 6th ed. Elsevier, Inc. 3251 Riverport Lane, St. Louis, Missouri 63043

Libbrecht, L., Valeer, D., Tania, R. 2005: Preneoplastic lesions in human hepatocarcinogenesis. Liver International 2005: 25: 16-27.

Massimo, P., Jose, M, 2014. Update on the pathophysiology of liver fibrosis Expert Review of Gastroenterology \& Hepatology Pages 459-472

Mousa, A., El-Gansh, H., Abd Eldaim, M., Mohamed, M., Morsi, A., El Sabagh, H. 2019: Protective effect of Moringa oleifera leaves ethanolic extract against thioacetamide-induced hepatotoxicity in rats via modulation of cellular antioxidant, apoptotic and inflammatory markers. Environmental Science and Pollution Research (2019) 26:32488-32504

Ramos-Tovar, E., Casas-Grajales, S., Hernández-Aquino, E., FloresBeltrán, R., Galindo-Góme, S., Vera-Aguilar, E., Diaz-Ruiz, A., Montes, S., Camacho, J., Tsutsumi, V., Muriel, P. 2019: Cirrhosis induced by thioacetamide is prevented by stevia. Molecular mechanisms. Journal of Functional Foods 52: 552-564

.Ramos-Vara, J., Kiupel, M., Baszler, T., Bliven, L., Brodersen, B., Chelack, B., West, K., Czub, S., Del Piero, F., Dial, S. 2008: Suggested guidelines for immunohistochemical techniques in 
veterinary diagnostic laboratories. Journal of Veterinary Diagnostic Investigation 20: 393-413.

Sanchez-Valle, V., Chavez-Tapia, N., Uribe, M., MendezSanchez, N. 2012: Role of Oxidative Stress and Molecular Changes in Liver Fibrosis . current Medicinal Chemistry, Volume 19, Number 28, 2012, pp. 4850-4860(11)

Youssef, M., Maghraby, H., Youssef, E. El-Sayed, M. 2012: Expression of Ki 67 in hepatocellular carcinoma induced by diethylnitrosamine in mice and its correlation with histopathological alterations. J. Appl. Pharm. Sci., 2(3):52-9.

Zargar, S. 2014: Protective effect of Trigonella foenum-graecum on thioacetamide induced hepatotoxicity in rats. Saudi journal of biological sciences 21: 139-145.

Zimmermann, H., Seidler, S., Gassler, N., Nattermann, J., Luedde, T., Trautwein, C., Tacke, F. 2011: Interleukin-8 is activated in patients with chronic liver diseases and associated with hepatic macrophage accumulation in human liver fibrosis. PLoS One 6: e21381 